\title{
Counterintuitive Alignment of $\mathbf{H}_{2}^{+}$in Intense Femtosecond Laser Fields
}

\author{
L. J. Frasinski,* J. Plumridge, J. H. Posthumus, ${ }^{\dagger}$ and K. Codling \\ J. J. Thomson Physical Laboratory, The University of Reading, Whiteknights, Reading RG6 6AF, United Kingdom
}

P. F. Taday, E. J. Divall, and A. J. Langley

Central Laser Facility, Rutherford Appleton Laboratory, Chilton, Didcot OX11 OQX, United Kingdom

(Received 3 August 2000)

\begin{abstract}
The multiphoton ionization of $\mathrm{H}_{2}$ has been studied using laser pulses of $266 \mathrm{~nm}$ wavelength, $250 \mathrm{fs}$ duration, and $5 \times 10^{13} \mathrm{~W} / \mathrm{cm}^{2}$ peak intensity. Dissociation of $\mathrm{H}_{2}{ }^{+}$via one-photon absorption proceeds through two channels with markedly different proton angular distributions. The lower-energy channel (2.6 eV kinetic energy release) is produced in the bond softening mechanism, which generates parallel alignment. The higher-energy channel $(3.5 \mathrm{eV})$ originates from population trapping in a light-induced bound state, where bond hardening generates orthogonal, counterintuitive alignment.
\end{abstract}

DOI: 10.1103/PhysRevLett.86.2541

Alignment is a clear example of spatial manipulation of molecules using intense lasers [1]. Despite the conceptual simplicity of inducing a dipole moment along the molecular axis and forcing it into an alignment with the laser $E$ field, the experimental evidence for this mechanism has a surprisingly checkered history. The earliest experiments showed that molecules multiply ionize and undergo Coulomb explosion when exposed to laser fields comparable to the intramolecular field [2]. A strong directionality of the fragment ions along the laser polarization direction was explained as a purely geometric effect-in an aligned molecule the field acts over a longer distance and the molecule is more efficiently ionized than at other angles [3]. That is, from a randomly oriented sample in the focal region, those molecules are ionized that are approximately aligned with the field and rapid axial recoil preserves their original orientation.

It was later pointed out that no ions were ejected in the direction orthogonal to the $E$ field [4,5], and it was argued that the geometric effect is too weak to explain this observation. It was concluded that even in the heavy $\mathrm{I}_{2}$ molecule a dynamic alignment must take place, or at least the laser must impart a substantial impulse to the fragments [4,5]. However, these ideas were formulated prior to the discovery of enhanced ionization at the critical internuclear separation [6-8]. As the molecule dissociates with about twice the equilibrium separation, the ionization threshold drops by about an order of magnitude if the molecule is parallel to the field but stays almost constant if the molecule is in an orthogonal orientation. It turns out that this enhancement is sufficient to explain the fragmentation anisotropy of $\mathrm{I}_{2}$ in purely geometric terms, but fails to reproduce the anisotropy of lighter molecules [9]. An elegant method of separating the dynamic alignment from the geometric effect using linear and circular laser polarization supports this view [10].

Our current understanding, then, is that in the dissociative ionization in intense femtosecond laser pulses, $\mathrm{H}_{2}$ molecules are strongly aligned, the medium-weight diatomics,
PACS numbers: $33.80 . \mathrm{Rv}, 42.50 . \mathrm{Hz}, 42.65 . \mathrm{Re}$

such as $\mathrm{N}_{2}$ or $\mathrm{Cl}_{2}$, are aligned to a lesser extent, but the heavy $I_{2}$ molecule is not. Normally, the alignment is parallel to the $E$ field, but in this Letter we present experimental evidence for orthogonal, counterintuitive alignment.

The Ti:sapphire laser system and ion time-of-flight apparatus used here have been described previously $[11,12]$. Briefly, laser pulses of $266 \mathrm{~nm}$ wavelength (third harmonic), $250 \mathrm{fs}$ duration, and $10 \mathrm{~Hz}$ repetition rate were focused in an ultrahigh vacuum chamber using a parabolic mirror with a focal length of $2 \mathrm{~cm}$, giving peak intensities up to $3 \times 10^{14} \mathrm{~W} / \mathrm{cm}^{2}$. The laser beam of $3 \mathrm{~mm}$ diameter was linearly polarized and the polarization plane could be rotated using a half-wave plate. Hydrogen gas was introduced via simple effusion and an external electric field directed fragment ions into a $13-\mathrm{cm}-$ long drift tube. Ions were detected by microchannel plates with a $10-\mathrm{mm}$ diameter aperture in front. Ion time-of-flight (TOF) spectra and pulse energies were recorded by a digital oscilloscope for each laser shot and for a range of half-wave plate positions, and stored in a computer.

Figure 1 shows ion TOF spectra recorded at a peak intensity of $5 \times 10^{13} \mathrm{~W} / \mathrm{cm}^{2}$ and four different angles, $\theta$, between the polarization direction and the drift tube axis. To compensate for a small intensity drift, the spectra were normalized to give the same height of the $\mathrm{H}_{2}{ }^{+}$peak and highlight amplitude changes in the $\mathrm{H}^{+}$peaks. Dissociation of $\mathrm{H}_{2}{ }^{+}$via one-photon absorption gives one "forward" and one "backward" $\mathrm{H}^{+}$peak, $1 \omega$, with about $3 \mathrm{eV}$ kinetic energy release (KER). The near-zero KER peak involves zero-photon dissociation (ZPD) [13], where no net number of photons is absorbed, despite energy being extracted from the laser field in a dynamic Raman process $[11,12]$.

The variation of the detector angular acceptance with the KER is plotted above the $\mathrm{H}^{+}$peaks. We see that the ZPD protons are collected from the full solid angle, and this explains the lack of variability of this peak with the polarization angle. The $1 \omega$ protons are collected from a small solid angle of $0.2 \mathrm{sr}$. This peak is strongest at $\theta=0^{\circ}$ and its KER appears to vary with $\theta$. However, detailed 


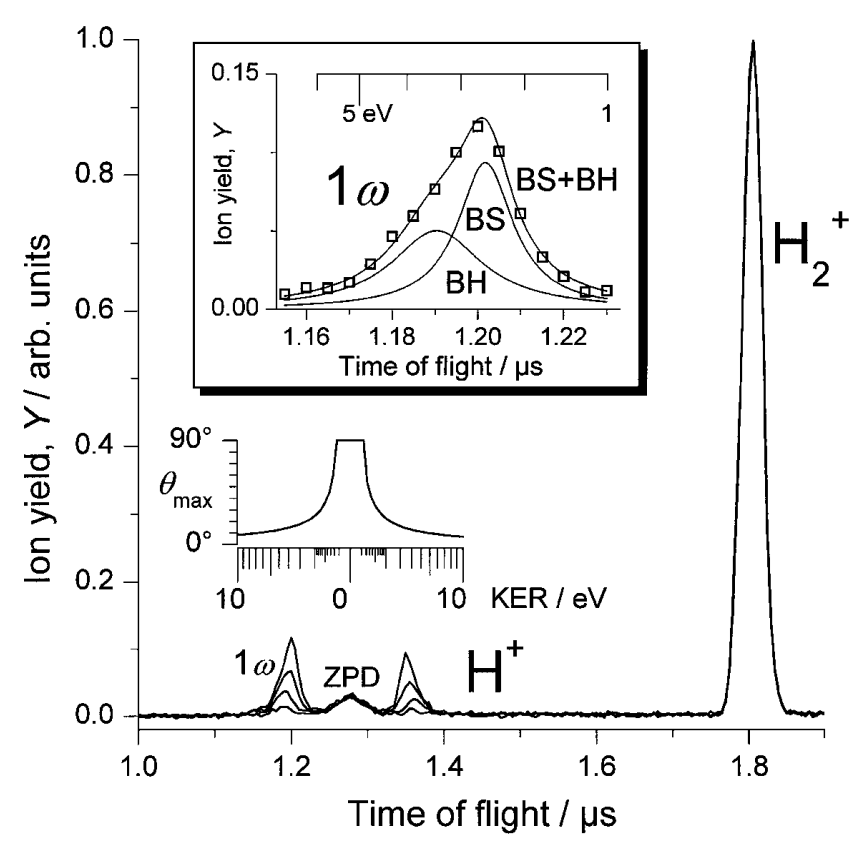

FIG. 1. Time-of-flight spectra of $\mathrm{H}_{2}$ exposed to laser pulses of $266 \mathrm{~nm}$ wavelength, 250 fs duration, $5 \times 10^{13} \mathrm{~W} / \mathrm{cm}^{2}$ peak intensity, at four angles, $\theta=0^{\circ}, 30^{\circ}, 60^{\circ}$, and $90^{\circ}$ (top to bottom), between the laser polarization and the drift tube axis. The scale above the $\mathrm{H}^{+}$peaks gives the kinetic energy release (shared between $\mathrm{H}_{\text {and }} \mathrm{H}^{+}$), and the bell curve shows the variation of the half-cone angle, $\theta_{\max }$, of the detector angular acceptance. The upper inset shows the one-photon absorption peak, $1 \omega$, at $\theta=0^{\circ}$ decomposed into bond-softening (BS) and bondhardening $(\mathrm{BH})$ dissociation channels of $\mathrm{H}_{2}{ }^{+}$. The zero-photon dissociation (ZPD) process involves no net number of photons absorbed and gives low-energy protons.

analysis reveals two channels of constant KER (peak energies 2.6 and $3.5 \mathrm{eV}$, as shown in the inset of Fig. 1), with different angular distributions, rather than a single channel with a variable KER. Two Lorentzian curves were fitted to the forward and backward peaks at 14 different angles, $\theta$, using $\chi^{2}$ minimization. The positions and widths of the Lorentzians were constrained to be the same for all angles but their amplitudes were allowed to vary. Figure 2 shows the variation of these amplitudes, with error bars corresponding to 1 standard deviation of the $\chi^{2}$ distribution.

The process of $\mathrm{H}_{2}{ }^{+}$dissociation in strong laser fields is most easily understood in terms of the lowest two $\mathrm{H}_{2}{ }^{+}$ potential curves dressed in the photon field [14]. Figure 3 shows the $1 s \sigma_{g}$ state dressed by a large number of photons, and the $2 p \sigma_{u}$ state with one photon less, which is absorbed from the field. At low laser intensities the two curves remain basically unperturbed, representing diabatic states crossing at the one-photon resonance (dashed lines). When the intensity increases, the curve crossing becomes an anticrossing and an energy gap steadily opens between the upper and lower branches of the new, adiabatic states (dot-dashed and solid lines). At an arbitrary angular position of the molecule, the width of the gap is determined

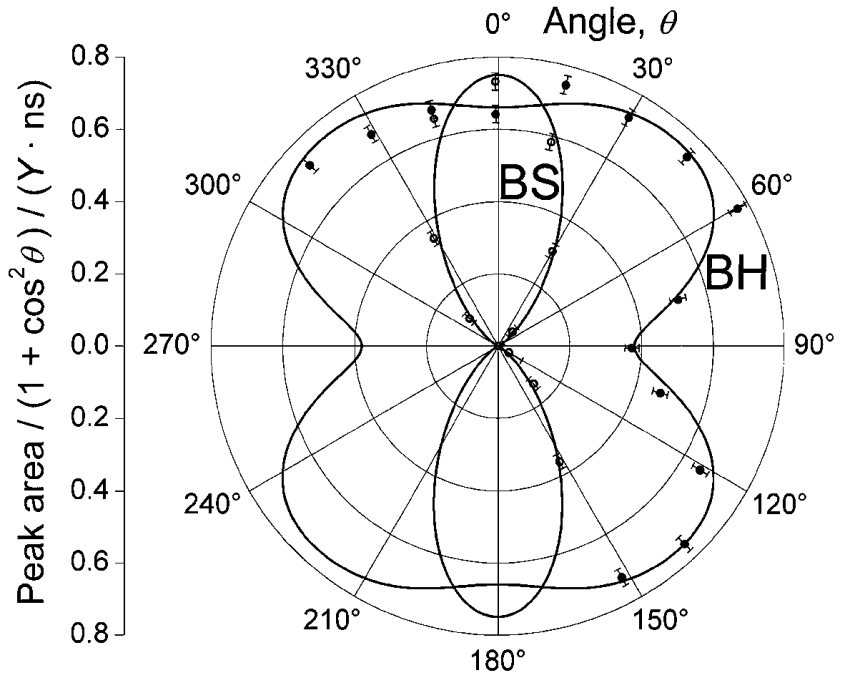

FIG. 2. Angular distributions of the BS and BH channels of Fig. 1 corrected for angular variation of ionization probability of neutral $\mathrm{H}_{2}$. The $\mathrm{BS}$ channel shows alignment along the laser $E$ field $\left(\theta=0^{\circ}\right)$. The BH channel reveals partial orthogonal alignment.

by the "parallel intensity" [15], $I \cos ^{2} \theta$. Therefore the gap can be opened not only by increasing the laser intensity, $I$, but also by rotating the molecule parallel to the $E$ field, as the values of $\theta$ indicate in Fig. 3.

The lower energy channel of $1 \omega$ is produced in the process of bond softening (BS) [16]. As the laser intensity increases, the $\mathrm{H}_{2}{ }^{+}$ion, initially bound in the $1 s \sigma_{g}$ potential well, can pass the gap (partially tunneling through the adiabatic barrier) and dissociate with the $1 \omega$ limit; see the lower path in Fig. 3. The peak energy of this channel $(2.6 \mathrm{eV})$ is $0.2 \mathrm{eV}$ below the center of the gap. This may simply reflect the higher population of lower vibrational levels, but more interestingly, may be the signature of an energy shift in a dynamic Raman effect, when the bond-softening branch of the anticrossing shifts down as the molecule dissociates.

The $\mathrm{H}_{2}{ }^{+}$population in the higher vibrational levels can be partially trapped in the process of bond hardening [11]. Classically, this means that a wave packet of a higher kinetic energy preferentially follows the diabatic route into the upper branch of the crossing. Increasing laser intensity progressively cuts off the return along this route and traps a substantial part of the wave packet above the onephoton gap; see the first part of the upper path in Fig. 3. This trapped population can now follow two different dissociation routes. A further increase of laser intensity lifts the wave packet and enables it to proceed to the $0 \omega$ limit in the ZPD process (the upper path). A reduction of the parallel intensity releases the wave packet from the trap, splitting it between the attractive and repulsive diabatic states. The part in the repulsive state immediately dissociates to the $1 \omega$ limit, as indicated. The remaining part 


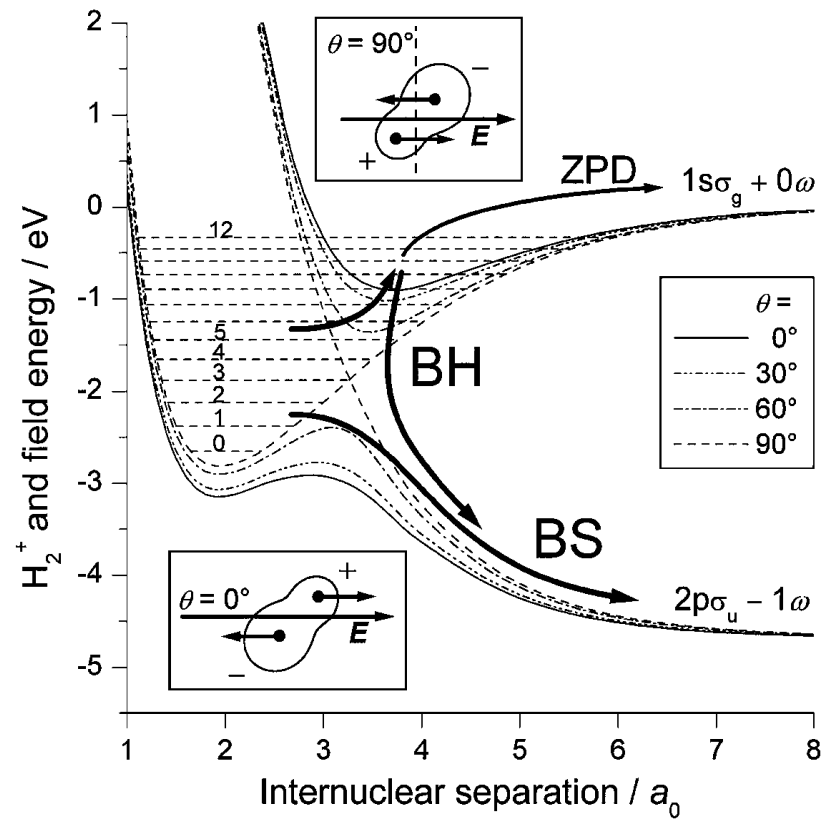

FIG. 3. Potential energy curves of $\mathrm{H}_{2}{ }^{+}$dressed in a photon field of $266 \mathrm{~nm}$ wavelength and $5 \times 10^{13} \mathrm{~W} / \mathrm{cm}^{2}$ intensity, calculated by Hamiltonian matrix diagonalization [15]. Lower vibrational levels dissociate via bond softening (BS), where the laser $E$ field effectively pulls the molecule into parallel alignment (lower box). Higher vibrational levels can be trapped in the bond-hardened $(\mathrm{BH})$ state on the upper branch of the anticrossing, where the $E$ field induces a counterintuitive, negative polarization and pushes the molecule into orthogonal alignment (upper box). This process is frustrated by the anticrossing gap shrinking as $\theta$ approaches $90^{\circ}$, whereupon the system falls onto the lower branch and the alignment is partially reversed.

of the released wave packet continues to oscillate in the $1 s \sigma_{g}$ potential well but still may have a significant probability of an adiabatic transition to the $1 \omega$ limit through the partially open gap.

The probability of diabatically crossing the gap depends mainly on the width of the gap and is given by the LandauZener formula [15]. For the trapping process outlined above, this probability is $50 \%$ when the gap half-width is $0.3 \mathrm{eV}$ and drops to $2 \%$ when the gap opens to $0.5 \mathrm{eV}$. Because the peak KER $(3.5 \mathrm{eV})$ of the second channel of the $1 \omega$ process is $0.7 \mathrm{eV}$ above the center of the gap, this strongly suggests that this channel experiences trapping in the bond-hardening $(\mathrm{BH})$ state above the gap.

In explaining the TOF spectra solely in terms of $\mathrm{H}_{2}{ }^{+}$dissociation, we have ignored the first ionization step-the $\mathrm{H}_{2}{ }^{+}$is produced from $\mathrm{H}_{2}$ by the same laser pulse. Fortunately, the two processes can be considered as strictly sequential, i.e., the ionization of $\mathrm{H}_{2}$ is followed by the dissociation of $\mathrm{H}_{2}{ }^{+}[12,14,17]$. This means that the fragment kinetic energies reflect the physics of the $\mathrm{H}_{2}{ }^{+}$ion, with the first ionization step simply preparing a certain distribution of $\mathrm{H}_{2}{ }^{+}$vibrational levels. However, the situation becomes more complicated if we wish to understand the fragment angular distributions with respect to the laser $E$ field, because any angular variation of the $\mathrm{H}_{2}$ ionization probability must be incorporated.

The Perelomov, Popov, and Terent'ev (PPT) theory, originally based on tunneling from hydrogenic atoms [18], gives reasonably accurate ionization probabilities for molecules on the assumption of spherical symmetry $[19,20]$. In modeling the true molecular shape, one needs to consider the angular variation of the potential barrier in tunnel ionization [3]. Numerical calculations have been performed recently [21] using an atomic barrier of effective charge, $Z_{\text {eff }}$, to approximate the molecular barrier in $\mathrm{D}_{2}$. We have repeated the modeling outlined in this paper and found that the maximum $Z_{\text {eff }}=1.05$ when $\mathrm{H}_{2}$ is parallel to the field $\left(\theta=0^{\circ}\right)$ and drops to $Z_{\text {eff }}=0.97$ when $\theta=90^{\circ}$, with a variation of less than $1 \%$ for the laser intensities of interest $\left[(0.3-10) \times 10^{13} \mathrm{~W} / \mathrm{cm}^{2}\right]$. Using the PPT formula we obtain an ionization probability for the parallel case that is almost exactly twice that for the perpendicular case, with a smooth variation for intermediate angles that is well approximated by $c(\theta)=1+\cos ^{2} \theta$.

We are well aware that $c(\theta)$ only takes into account tunneling at the equilibrium internuclear separation and that multiphoton resonances [22] or bond softening in neutral $\mathrm{H}_{2}$ [23] would modify this simple model. However, in the absence of further information, we feel it is better to incorporate $c(\theta)$ rather than ignore it. Therefore, we have divided the TOF peak areas of Fig. 1 by $c(\theta)$ to arrive at the angular distributions of $\mathrm{H}^{+}$shown in Fig. 2, which now represent the behavior of an initially isotropic $\mathrm{H}_{2}{ }^{+}$ensemble. We approximate the BS and $\mathrm{BH}$ channels, respectively, with analytical curves given by the expressions $0.75 \cos ^{6} \theta$ and $0.76-0.10 \cos ^{12} \theta-$ $0.38 \sin ^{12} \theta$ (solid lines). While some points deviate from these curves, the angular resolution of the detector does not justify introducing more harmonics.

The sharp peaking of the BS channel along the laser field can be understood in classical terms. The laser field polarizes the molecule (the electronic charge oscillates in phase with the laser field) and exerts a torque that aligns the molecule parallel to the field (see the lower box in Fig. 3).

The BH channel displays a counterintuitive effect that has a quantum mechanical origin. On the upper, bondhardening branch of the anticrossing, the diabatic $1 s \sigma_{g}$ and $2 p \sigma_{u}$ wave functions superpose in such a way that the polarization is negative, i.e., the electronic charge oscillates in antiphase with the laser field. The negative polarization produces a torque that starts to align the molecule orthogonally to the field (see the upper box in Fig. 3). However, the completion of this alignment is frustrated by a reducing energy gap as $\theta$ approaches $90^{\circ}$ and an increased rate of diabatic transitions to the lower branch of the anticrossing; consequently, the alignment is partially reversed. The overall effect is peaking of the $\mathrm{BH}$ channel at intermediate angles, reflecting the two opposing processes of alignment. 
Rather than considering forces acting on the molecule, one can study potential energy surfaces to explain the alignment dynamics. The anticrossing curves of Fig. 3 create two such surfaces that are functions of internuclear separation and angular position [15,24]. On either of these surfaces the system seeks a minimum. On the BS surface this corresponds to the dissociation path of maximal ac Stark shift, along the $0^{\circ}$ direction. On the BH surface the energy minimum is at $90^{\circ}$, where the Stark shift is minimal. However, this minimum is leaky and the system falls down to the BS surface.

A deviation from parallel alignment was predicted in an early theoretical paper on $\mathrm{Ar}_{2}{ }^{+}$undergoing bond hardening [25]. The salient features of Fig. 2 are reproduced by calculations of bond hardening in $\mathrm{H}_{2}{ }^{+}$exposed to laser pulses of $213 \mathrm{~nm}$ wavelength and $100 \mathrm{fs}$ pulse duration (see Fig. 6 of Ref. [26]). The calculations show that the peak of the angular distributions shifts to higher angles $\left(\theta=30^{\circ} \rightarrow 60^{\circ}\right)$ for increasing vibrational quantum number $(\nu=4 \rightarrow 6)$, which reflects more efficient trapping of higher vibrational levels, i.e., a shift from the BS to the BH channel in our Fig. 2.

The contribution of geometric, parallel, and orthogonal alignment has been explicitly calculated for the ground rovibrational level of $\mathrm{H}_{2}{ }^{+}$exposed to 80 and $160 \mathrm{~nm}$ femtosecond laser pulses [27]. Comparing models of fixed and floating molecular axes it has been shown that the minimum of ionization probability at $\theta=90^{\circ}$ is mainly due to the geometric effect, while the lobe structure from $0^{\circ}$ to about $60^{\circ}$ reflects a dynamic interplay of the parallel and orthogonal alignment, the latter being stronger at the shorter wavelength. Indeed, short wavelength seems to be essential to study orthogonal alignment. Qualitatively we observe similar angular distributions for the $1 \omega$ peak using the second harmonic at $400 \mathrm{~nm}$. However, a quantitative analysis of these data is hampered by weaker $\mathrm{BH}$ and $\mathrm{ZPD}$ channels, reflecting a smaller population of vibrational levels that can be trapped above the one-photon crossing at longer wavelengths [12].

We are pleased to acknowledge the Engineering and Physical Sciences Research Council (U.K.) for their financial support.

Note added.-After this work was submitted, Talebpour, Vijayalakshmi, Bandrauk, Nguyen-Dang, and Chin [28] have reported a perpendicular ejection of $\mathrm{D}^{+}$ions explaining it in terms of multiphoton transitions to higher excited states of $\mathrm{D}_{2}{ }^{+}$.

*Email address: L.J.Frasinski@reading.ac.uk †Present address: Sektion Physik, University of Munich, Am Coulombwall 1, 85748 Garching, Germany.

[1] P. B. Corkum, C. Ellert, M. Mehendale, P. Dietrich, S. Hankin, S. Aseyev, D. Rayner, and D. Villeneuve, Faraday Discuss. 113, 47 (1999).
[2] L. J. Frasinski, K. Codling, P. A. Hatherly, J. Barr, I. N. Ross, and W. T. Toner, Phys. Rev. Lett. 58, 2424 (1987).

[3] K. Codling, L. J. Frasinski, and P. A. Hatherly, J. Phys. B 22, L321 (1989).

[4] D. T. Strickland, Y. Beaudoin, P. Dietrich, and P. B. Corkum, Phys. Rev. Lett. 68, 2755 (1992).

[5] D. Normand, L. A. Lompré, and C. Cornaggia, J. Phys. B 25, L497 (1992).

[6] J. H. Posthumus, L. J. Frasinski, A. J. Giles, and K. Codling, J. Phys. B 28, L349 (1995).

[7] T. Seideman, M. Yu. Ivanov, and P. B. Corkum, Phys. Rev. Lett. 75, 2819 (1995).

[8] T. Zuo and A.D. Bandrauk, Phys. Rev. A 52, R2511 (1995).

[9] J. H. Posthumus, J. Plumridge, M. K. Thomas, K. Codling, L. J. Frasinski, A. J. Langley, and P. F. Taday, J. Phys. B 31, L553 (1998); 31, L985 (1998).

[10] Ch. Ellert and P. B. Corkum, Phys. Rev. A 59, R3170 (1999).

[11] L. J. Frasinski, J. H. Posthumus, J. Plumridge, K. Codling, P. F. Taday, and A. J. Langley, Phys. Rev. Lett. 83, 3625 (1999).

[12] J. H. Posthumus, J. Plumridge, L. J. Frasinski, K. Codling, E. J. Divall, A. J. Langley, and P. F. Taday, J. Phys. B 33, L563 (2000).

[13] A. Giusti-Suzor and F. H. Mies, Phys. Rev. Lett. 68, 3869 (1992).

[14] A. Giusti-Suzor, F. H. Mies, L.F. DiMauro, E. Charron, and B. Yang, J. Phys. B 28, 309 (1995).

[15] A. Zavriyev, P. H. Bucksbaum, H. G. Muller, and D. W. Schumacher, Phys. Rev. A 42, 5500 (1990).

[16] A. D. Bandrauk and M. L. Sink, J. Chem. Phys. 74, 1110 (1981).

[17] M. R. Thompson, M. K. Thomas, P.F. Taday, J. H. Posthumus, A. J. Langley, L. J. Frasinski, and K. Codling, J. Phys. B 30, 5755 (1997).

[18] A. M. Perelomov, V. S. Popov, and M. V. Terent'ev, Sov. Phys. JETP 23, 924 (1966).

[19] P. Dietrich and P. B. Corkum, J. Chem. Phys. 97, 3187 (1992).

[20] P. Dietrich, M. Yu. Ivanov, F. A. Ilkov, and P. B. Corkum, Phys. Rev. Lett. 77, 4150 (1996).

[21] A. Talebpour, S. Larochelle, and S. L. Chin, J. Phys. B 31, L49 (1998).

[22] S. W. Allendorf and A. Szöke, Phys. Rev. A 44, 518 (1991).

[23] I. Kawata, H. Kono, Y. Fujimura, and A. D. Bandrauk, Phys. Rev. A 62, 031401 (2000).

[24] R. Numico, A. Keller, and O. Atabek, Phys. Rev. A 60, 406 (1999).

[25] A. D. Bandrauk and G. Turcotte, J. Phys. Chem. 87, 5098 (1983).

[26] E. E. Aubanel, J.-M. Gauthier, and A. D. Bandrauk, Phys. Rev. A 48, 2145 (1993).

[27] R. Numico, A. Keller, and O. Atabek, Phys. Rev. A 52, 1298 (1995).

[28] A. Talebpour, K. Vijayalakshmi, A. D. Bandrauk, T. T. Nguyen-Dang, and S. L. Chin, Phys. Rev. A 62, 042708 (2000). 